\title{
The Precision Medicine Initiative's All of Us Research Program: an agenda for research on its ethical, legal, and social issues
}

\author{
Pamela L. Sankar, PhD ${ }^{1}$ and Lisa S. Parker, $\mathrm{PhD}^{2}$
}

The Precision Medicine Initiative (PMI) is an innovative approach to developing a new model of health care that takes into account individual differences in people's genes, environments, and lifestyles. A cornerstone of the initiative is the PMI All of Us Research Program (formerly known as PMI-Cohort Program), which will create a cohort of 1 million volunteers who will contribute their health data and biospecimens to a centralized national database to support precision medicine research. The PMI All of US Research Program is the largest longitudinal study in the history of the United States. The designers of the Program anticipated and addressed some of the ethical, legal, and social issues (ELSI) associated with the initiative. To date, however, there is no plan to call for research regarding ELSI associated with the PMI All of Us Program. Based on analysis of National Institutes of Health (NIH) funding announcements for the PMI All of Us
Program, we have identified three ELSI themes: cohort diversity and health disparities, participant engagement, and privacy and security. We review All of Us Research Program plans to address these issues and then identify additional ELSI within each domain that warrant ongoing investigation as the All of Us Research Program develops. We conclude that PMI's All of Us Research Program represents a significant opportunity and obligation to identify, analyze, and respond to ELSI, and we call on the PMI to initiate a research program capable of taking on these challenges.

Genet Med advance online publication 8 December 2016

Key Words: diversity; ELSI; mHealth; precision medicine; privacy
In January 2015, President Obama announced the Precision Medicine Initiative (PMI), a major program to develop the nationwide infrastructure necessary to implement precision medicine in the United States. A cornerstone of the PMI is the creation of a cohort of 1 million volunteers. Enrollment is expected to begin in late 2016, marking the launch of the largest longitudinal study in the history of the United States. In the PMI All of Us Research Program (PMI-AURP), previously called the PMI Cohort Program, volunteers will contribute their health data and biospecimens to a centralized national database, providing a platform for biomedical and behavioral research that can identify individual differences in disease etiology and course by examining variability in genes, environment, and lifestyle. ${ }^{1}$ In addition to the cohort itself, this platform will include a comprehensive, complex information technology (IT) infrastructure that will be the vehicle for collecting, storing, analyzing, and sharing patient data. The knowledge produced through research employing this platform is expected to overcome limitations of current healthcare models that are based on treating a "typical patient" 2 and thus ignore the potential for individual variation, as well as to have a "transformative" effect on "how we understand-and how we approach-the health and wellness of families, communities, and individuals." 3

Several reviews have identified ethical, legal, and social issues (ELSI) associated with the paradigm shift that precision medicine represents. ${ }^{4-12}$ Designers of the PMI-AURP have anticipated and begun to address some of the ELSI associated with the initiative, for example, by requiring strict privacy and security protections and implementing an extensive program for participant engagement. To date, however, there is no explicit plan to call for research regarding ELSI associated with the design and implementation of the PMI-AURP. In this regard, the PMI-AURP differs from many large genomics-related NIH initiatives, such as the Human Microbiome Project, the Clinical Sequencing Exploratory Research program, the Electronic Medical Records and Genomics (eMERGE) Network, and the Human Genome Project (HGP) itself, which include robust research programs to examine ELSI likely to arise both in the initiatives themselves and in the broader and longer-term implications of accomplishment of their goals.

Important features of the PMI-AURP demonstrate the need for ELSI research. First, implementation of PMI-AURP entails significant changes to the relationship between patients and

${ }^{1}$ Department of Medical Ethics and Health Policy, Perelman School of Medicine, University of Pennsylvania, Philadelphia, Pennsylvania, USA; ${ }^{2}$ Department of Human Genetics, Center for Bioethics \& Health Law, University of Pittsburgh, Pittsburgh, Pennsylvania, USA. Correspondence: Pamela L. Sankar (sankarp@upenn.edu) 
their health care, which are evident in the new demands for patient information. PMI-AURP participants must be willing to contribute data freely, generously, regularly, and longitudinally, including (i) agreeing to ongoing accessibility of their electronic health records, (ii) participating in and sharing the results of additional clinical and behavioral assessments, (iii) contributing DNA samples and other biologic specimens, and (iv) participating in mobile health (mHealth) data-gathering activities to collect geospatial and environmental data. These data will be made available for research to academic and commercial researchers and to citizen scientists; their findings, once tested and confirmed, will be integrated to improve care and used to drive more research. The long term objective is better healthcare for everyone, but the means of achieving it includes reconceptualizing PMI-AURP particpants' health information, traditionally considered intensely personal, as a social good. For the group to benefit, individuals must contribute.

Convincing individuals to reconceptualize the purpose of their health information in this way requires building and maintaining public trust. The NIH faced a similar challenge at the inception of the HGP. In an exceptional move, it chose to gain that trust by making a commitment to support research to identify and address ELSI raised by the HGP. For PMI-AURP to establish and warrant public trust, it must make the same commitment to identify and address ELSI associated with the operations and research of PMI-AURP. This is particularly important to help ensure the participation of racial and ethnic minorities and medically underserved populations who continue to demonstrate varying levels of trust in health-care providers and researchers. ${ }^{13-15}$

Second, there are compelling advantages to studying the PMIAURP from its inception. Many of the technologies and practices required by PMI-AURP to fulfill its mandates still need to be developed, including the IT system and protocols governing basic practices such as returning results to participants. Others are in their nascency, such as mHealth technologies and participatory health care through individuals' access to and utilization of information in their electronic health records. In the early days of the PMI-AURP critical decisions will be made that will shape the trajectory not only of the program itself but also of precision medicine for years to come. Therefore, early findings from ELSI research initiated in partnership with the scientific programs could be used to inform ongoing governance, policies, practices, and evaluation of PMI-AURP as well the development of empirically informed normative frameworks for the future practice of precision medicine. An example of such potential contributions might involve a protocol to randomly assign and study different methods for the return of results to cohort members. In addition, the reliance of the PMI-AURP on novel technologies, applied on a scale not previously witnessed and never systematically studied, suggests that examining associated ELSI is critical and will have relevance beyond the domain of precision medicine. The PMI-AURP provides an unprecedented opportunity to conduct research on the ELSI associated with precision medicine that will have implications that extend well beyond this project. Without the initiation of a ELSI research program, this opportunity could be lost. For this reason, we call on the PMI to initiate a robust ELSI research program at the inception of the project.

In this article, we identify ELSI likely to emerge as the PMIAURP is implemented. We base our description of PMI-AURP on NIH funding announcements issued in November 2015 to solicit proposals for the project's component programs. These funding announcements present the NIH's vision for the PMIAURP and are the most detailed official descriptions currently available. Table 1 shows the components of PMI-AURP, the associated funding announcement, a description of the component's function, and the institutions chosen to implement each component. Readers should consult the PMI-AURP website for ongoing updates regarding the program's structure and implementation (https://www.nih.gov/research-training/ allofus-research-program).

The six funding announcements for the PMI-AURP outline a multipart program, a major feature of which is a network of health-care-provider organizations (HPOs) that will be responsible for recruiting the majority of participants. Other participants will join through the Direct Volunteers program. The HPOs will manage health data and biospecimen collection and be responsible for ongoing participant engagement and retention. Support for their work will be provided by the PMI-AURP Biobank, which will develop standards and protocols for specimen collection that will be stored and made available for research; a Communications Support program, which will centralize development of project messages and visual materials; and a Participants Technology Center (PTC), which will develop and help implement innovative mHealth technologies to facilitate data collection and patient engagement. In collaboration with the HPOs, a Coordinating Center (CC) will oversee management and coordination of the program's components.

\section{ELSI ASSOCIATED WITH THE PMI-AURP: ENVISIONED AND IN NEED OF STUDY}

A close reading of the funding announcements reveals three themes that appear in all the announcements and that are integral to the goals of the PMI-AURP: (i) cohort diversity and health disparities, (ii) participant engagement, and (iii) privacy and security. Although health disparities do not appear as a topic in the funding announcements, this issue relates directly to cohort diversity and is discussed here in that context. These themes reflect domains of ELSI research and provide a serviceable framework for examining potential ELSI related to PMI-AURP.

We briefly review the importance of each of the three themes with respect to PMI-AURP and note how the funding announcements articulate related ELSI. We then identify additional ELSI within each domain that warrant ongoing investigation as the PMI-AURP is being implemented. 
Table 1 PMI-AURP components: funding announcement and awarded institutions

\begin{tabular}{|c|c|c|}
\hline $\begin{array}{l}\text { PMI-AURP component and funding } \\
\text { announcement }\end{array}$ & Component description & Awarded institution \\
\hline $\begin{array}{l}\text { OT-PM-16-001; Precision Medicine } \\
\text { Initiative Cohort Program Direct Volunteers } \\
\text { Pilot Studies }\end{array}$ & $\begin{array}{l}\text { Pilot projects to jump-start PMI-AURP enrollment through direct } \\
\text { volunteers }\end{array}$ & Vanderbilt University \\
\hline $\begin{array}{l}\text { OT-PM-16-002; Communication Support } \\
\text { for the Precision Medicine Initiative } \\
\text { Research Programs at the NIH }\end{array}$ & $\begin{array}{l}\text { Develop project messages and visual materials; oversee testing } \\
\text { and dissemination }\end{array}$ & $\begin{array}{l}\text { Hungry Heart Media, Inc. (Wondros); } \\
\text { HCM Strategists, LLC }\end{array}$ \\
\hline $\begin{array}{l}\text { RFA-PM-16-002; Precision Medicine } \\
\text { Initiative Cohort Program Healthcare } \\
\text { Provider Organization Enrollment Centers } \\
\text { (UG3/UH3) }\end{array}$ & $\begin{array}{l}\text { Funds several groups to create a network of HPOs that will be } \\
\text { responsible for recruiting participants and explaining the project } \\
\text { to them. These HPOs will manage health data and biospecimen } \\
\text { collection and be responsible for ongoing participant } \\
\text { engagement and retention. }\end{array}$ & $\begin{array}{l}\text { Columbia University, New York } \\
\text { City, NY; Northwestern University, } \\
\text { Chicago, IL; University of Arizona, } \\
\text { Tucson, AZ; University of Pittsburgh, } \\
\text { Pittsburgh, PA }\end{array}$ \\
\hline $\begin{array}{l}\text { RFA-PM 16-004; Precision Medicine } \\
\text { Initiative Cohort Program Biobank (U24) }\end{array}$ & $\begin{array}{l}\text { Responsible for developing standards and protocols for } \\
\text { collection of specimens that will be stored and made available } \\
\text { for research }\end{array}$ & Mayo Clinic, Rochester, MN \\
\hline
\end{tabular}

HPO, health-care-provider organizations; NIH, National Institutes of Health; OT, Other Transaction; PMI-AURP, Precision Medicine Initiative All of Us Research Program.

${ }^{\text {aAs }}$ a result of changes made during the funding phase of the PMI-AURP, the HPOs will also officially take on some Coordinating Center responsibilities.

\section{Cohort diversity and health disparities}

Funding announcements outline plans to enroll a cohort that reflects diversity in many respects, including socioeconomic status, age, geography, health status, health literacy, and personal competence with information technologies. Plans also cite the need to include traditionally underserved populations, people with cognitive impairments, and families with children. ${ }^{16-18}$ In addition, particular emphasis is placed on the need to prioritize enrollment of racial and ethnic minorities, which the Precision Medicine Working Group report argues is vital for effective delivery of the benefits of precision medicine. ${ }^{1}$ The report explains that knowledge of genetic variation in different ancestry groups is necessary to identify differences in disease etiology and course, and that because self-identified race and ethnicity correlate with genetic variation, enrolling a racially and ethnically diverse population should have the effect of enrolling people from diverse genetic ancestries. ${ }^{1}$

The HPO funding announcement gave applicants latitude to decide specifically how they would meet the diversity requirement, assuming that the populations they chose helped to meet the PMI-AURP goal of reflecting the "rich diversity" of the United States. ${ }^{17}$ Applicants were asked to demonstrate that they planned to enroll "sufficient numbers" from these populations to be able to successfully "address scientific questions," and that they had previous success working with these populations. HPOs have been funded initially for only 1 year, pending demonstration that they can achieve the enrollments to which they committed. Subsequently, HPOs will monitor and periodically report their progress meeting projected enrollments.

Recruiting, enrolling, and retaining members of diverse populations who, as the HPO review requirements stipulated, "are engaged as partners in a longitudinal, long-term effort to transform the understanding of factors contributing to individual health"17 are challenging tasks. Support from the PTC and the Communications Support program, such as furnishing project materials in Spanish as well as English, is intended to help in this endeavor. Mandated ongoing evaluations to determine which outreach strategies seem to work best under different conditions, overseen by the CC, are also planned to help achieve and retain a diverse cohort.

Following the PMI-AURP's directions to create and maintain a cohort that reflects the diversity of the United States raises many questions beyond those that PMI-AURP plans have anticipated. This is particularly important because of the Program's potential implications for health-care disparities. Possible ELSI research questions include the following:

- How should the PMI-AURP assess whether the strategy of enrolling a diverse population based on racial, ethnic, and socioeconomic factors results in a cohort with the necessary variability to conduct research that has the potential to benefit traditionally underserved populations?

- If answering scientific questions requires oversampling or targeted enrollment of particular populations, then how will this targeting or oversampling be viewed by individuals from those populations? How might targeting be viewed if associated, for example, with a medical condition or continental ancestry? Is this a potential source of stigma for individuals or communities enrolled in PMI-AURP?

- How should such targeted enrollment be conducted? How should research results associated with particular 
ancestry groups be reported and utilized to avoid conflating social conceptualizations of race with genetic determinism?

- To achieve and maintain cohort diversity, will there be caps on the number of participants from certain groups who can enroll in the PMI-AURP and, if so, how will those caps be established and implemented? What consequences might caps have for participants' relationships with the HPO, their individual providers, or the PMIAURP overall?

Once enrolled, how do PMI-AURP participants experience the program and how do their experiences and perceptions vary over time and across different groups? Examining these questions might help explain what influences trends in PMIAURP membership over time. For example:

- What ramifications might the Program's reliance on computer-mediated social media tools have for health-care disparities? Will the elderly, poor, or disabled be less able to participate in and perhaps benefit from some PMIAURP activities because of lack of experience with social media or lack of access to the needed equipment (e.g., smart phones, data plans)?

- Will certain groups, such as rural residents, the elderly, families with young children, or the cognitively impaired, require support to facilitate participation, such as assistance with travel to the HPO?

- Will concerns about the government's role in data collection and management or about data-security breaches vary among different communities? Could these concerns shape PMI-AURP participation in a way that undermines generalizability of research findings to the broad American population?

- Will some populations be better able to take advantage of the data, results, and prevention recommendations likely to result from PMI-AURP research? How can the PMIAURP address such differences to avoid exacerbating health-care disparities?

- Will treatments or other health-care advances that might result from PMI-AURP research benefit some populations more than others? For example, will the eventual commercial use of PMI-AURP research findings concentrate on applications believed to be more marketable because they will apply to groups known to have greater economic resources? If some populations stand to benefit more or more immediately, then how should this disparity be ameliorated?

\section{Participant engagement}

In the PMI-AURP funding announcements, activities described as participant engagement range from membership in highlevel governance committees to acting as subjects in tests of mHealth data-collection protocols. Ongoing engagement activities that involve the largest number of participants will be overseen by the CC and conducted through the HPOs. The HPOs will recruit and enroll participants and work with them over the course of their involvement in the PMI-AURP. The PTC will contribute by developing the technology used for certain engagement activities. Preliminary work on patient engagement, such as creating a prototype informational website for participants, has started at Vanderbilt University under the auspices of pilot funding to the Direct Volunteer Program.

PMI-AURP planners characterize participant engagement as a fundamental feature of the PMI-AURP and discuss it in all the funding announcements. Elsewhere, they caution that this "highly interactive participation model...is untested for a project of this scale."16 This implies that PMI-AURP planners recognize that the types and uses of patient engagement may change over the course of the PMI-AURP. It seems likely that patient engagement activities in the early stage of the PMI-AURP will focus on creating policies and procedures that are necessary to get the program off the ground. A planned consultation with participants about policies for return of results is one such early task; similar tasks are likely to emerge over the course of the project. ${ }^{19}$ The NIH also envisions a more fundamental role for participant engagement. As the project matures, participant engagement is intended to "create durable relationships with volunteers" that will eventually "transform patients into partners"3 and thus help to usher in a new model of health care in which patients both actively manage their own health and help to raise and answer research questions.

To lay the foundation for this transformation, the CC, HPOs, and PTC are all directed to develop patient engagement plans that detail approaches for "partnering with research participants and their advocates in the governance, design, conduct, oversight, dissemination, and evaluation activities" and that comply with key principles informing the PMI-AURP. ${ }^{16-18}$ One key principle is an "inclusive philosophy of engagement" that enables participation by the populations targeted to achieve cohort diversity. In addition, HPOs must establish a participant retention plan and regularly report on its success; there is an expectation of assistance from the CC should they need to improve their performance.

Although PMI-AURP planning documents express a serious commitment to fostering patient engagement, they do not address its foundation or substance. Participant engagement can refer to a range of activities that vary in form, content, and intensity. There is as yet no common understanding of best practices for patient engagement. Mandated PMI-AURP research would seek to help fill this gap by developing "quantitative approaches" to determine which strategies "most effectively engage participants, particularly those historically underrepresented in biomedical research."17 However, research also suggests that one of the most important factors influencing the success of patient engagement is a common vision among volunteers and conveners of an engagement's objectives, or at least a common language with which to discuss their differences ${ }^{20-23}$ This suggests that ELSI research 
could contribute to the success of the PMI-AURP by examining key concepts that inform its participant engagement activities and asking how an understanding of them varies over time and across groups. A preliminary list of questions to consider includes the following:

- What is patient engagement and what purposes do its different forms serve?

- What are the benefits and burdens of patient engagement?

- What is the meaning of partnership?

- What is the meaning of health and well-being?

- What is the meaning and value of privacy?

Interest in elucidating foundational aspects of participant engagement could be advanced by deciding which principles should inform the process beyond inclusivity, which the funding announcements emphasize. Examples of ELSI that research might pursue include the following:

- What is the moral basis for asking participants to shoulder the burdens or enjoy the possible benefits of engagement?

- How should participants be selected for various forms of engagement? Criteria might include expertise, diversity, equity, inclusiveness, or convenience. On what moral basis should decisions regarding such criteria be made?

- What are the ethical and social implications of developing marketing materials centrally, rather than locally, for site-specific populations? How do potential participant populations respond to these centrally developed materials? Are there implications for recruitment of a diverse cohort? Does the use of centrally developed marketing and informed consent materials reflect a changing understanding of the normative relationship between institutions engaging in research activities (e.g., HPOs) and potential research participants?

- What should be the process for assessing possible conflicts of interest in participant engagement, particularly when participants have a role in governance? Should individuals representing disease conditions be encouraged or limited in participation, based on prevalence or other characteristics? Alternatively, should the input and participation of individuals with conditions of great public health priority be specifically encouraged?

- Under what conditions might it be appropriate to remove participants from various aspects of the PMI-AURP (e.g., from governance activities or from participation in data collection)?

The envisioned transformation of individuals from research participants into partners in research and their own health care raises questions about participants' rights and responsibilities.

- Having agreed to join the PMI-AURP, what are participants' moral obligations to actively cooperate with engagement activities? Does participant perception of this obligation differ from investigators' or planner's perceptions, or does it vary across participant groups?

- How will disagreements between participants and researchers be settled? For example, what if participants argue that research and interventions utilizing social media have potential harms or are a low priority? Would engagement on this issue change the data-collection plan? Should there be venues for participant complaints about the conduct of engagement activities or the progress of PMI-AURP overall?

- Will a phenomenon of "participant fatigue" be associated with continued long-term involvement? What might account for this-for example, the amount or type of engagement or, perhaps, the kinds of decisions being discussed?

- Within groups or communities, will leaders or those who speak for others emerge organically? What is the moral foundation for those parties to speak on behalf of others? Should participants have a voice in choosing which participants represent them in governance activities?

- Are there limits to participant engagement? Are there questions or issues that participant engagement cannot or should not address?

\section{Security and privacy}

The Precision Medicine Working Group report states that "maintaining robust privacy protection" is "essential" to the program's success. ${ }^{1}$ However, balancing the need to protect the privacy of participant information -including results of DNA analysis, electronic health record -derived data, results from behavioral assessments, and mHealth data - against the need to facilitate researchers' access to information poses a serious challenge for planners of the PMI-AURP and participating organizations. Complicating this balancing act is PMI-AURP's goal of feeding study results back into the system to guide both development of new treatments and individual participants' clinical care.

Security and privacy are discussed in all the PMI-AURP funding announcements. The CC is generally responsible for establishing security and privacy procedures for the PMI-AURP. Its funding announcement demands creation of a reliable, comprehensive, state-of-the-art IT security system, supported by strong institutional policies governing access and use. The system must comply with several sets of stringent federal security regulations, or their equivalent, and will be designed to ensure data security, with limited data access to authorized users for authorized purposes and to fulfill the privacy goal of ensuring that personal data, once de-identified, cannot be re-identified. ${ }^{16}$ Nonetheless, PMI-AURP documents concede that, best efforts notwithstanding, data breaches are possible; therefore, PMIAURP-funded organizations are mandated to develop procedures to immediately inform participants if breaches do occur. ${ }^{17}$

Considering the extensive demands and novel uses of participants' health information by the PMI-AURP, security and 
privacy concerns are likely to extend beyond those that can be handled by program policies and technical IT safeguards. No existing database of this size collects and combines the range of data types that the PMI-AURP intends to gather. mHealth data alone raise important questions. Described as the "digital traces in participants' daily lives," collected from "social media, phone usage, cable TV, automobiles, customer loyalty cards, home-based monitoring systems, wireless weight scales, and specially deployed home-based monitoring devices,"18 mHealth data are unlike traditional health information with regard to the mode and ubiquity of the surveillance activity collecting it. ELSI might arise from novel qualities of these data or from the aggregation of the PMI-AURP's more traditional data types. Specific ELSI concerns might include the following:

- What ELSI are presented by the government's collection of data that more closely resembles the data that nonhealth (e.g., security, policing, or commercial) entities have typically collected? How will the public perceive the collection of such data with potential use for health and non-health surveillance?

- What firewalls should exist between the PMI-AURP and government agencies determining eligibility for health care or other services? For example, what guarantees, if any, should be made to recipients of Medicaid and Medicare or disabled recipients of Supplemental Security Income that information collected by mHealth devices will not be used to deny services or eligibility?

- Might non-health data, when combined with detailed health data from multiple sources, yield unanticipated revelations about individual participants or groups? Considering the challenge of forecasting these eventualities, how should the potential privacy risks associated with these types of data be explained during the informed consent process?

- Does the integration of mHealth data raise special questions regarding access by parents or guardians to $\mathrm{mHealth}$ data for children or cognitively impaired adults or in other contexts?

- How should PMI-AURP data breaches be handled (beyond immediately notifying affected participants) to minimize harm to participants and in recognition of the risks associated with volunteering to participate in an experimental program?

- Do legal mechanisms exist or do they need to be created to ensure PMI-AURP accountability to specific individuals, groups, or the whole society? How does the traditional legal concept of sovereign immunity, or even the public's perception that the government is immune from civil and criminal liability, affect such accountability, perception of accountability, and recruitment efforts?

The distinct nature of PMI-AURP data also raises questions about its possible uses, including, for example:
- Which, if any, non-health-related requests for access to aggregate, de-identified PMI-AURP data should be entertained, and how will requests be adjudicated? For example, for policy-making purposes, should the use of PMI-AURP data be limited to development of policies for the protection of human health, or should other types of policies be informed by this public resource, such as environmental, agricultural, or transportation policies or legal regulations, including those concerning forensic identification or sovereignty issues related to Native American groups?

- Given that the Genetic Information Nondiscrimination Act of 2008 addresses only the use of genetic information, and that the Patient Protection and Affordable Care Act addresses individuals' preexisting health conditions, how should the use of environmental information (for example, by health insurers) be understood and regulated, particularly since insurers identify populations for coverage geographically and are permitted to engage in community rating in establishing premiums?

- The PMI-AURP specifically envisions citizen scientists utilizing the Program's data and infrastructure and contributing to the Program's database and research results. How should the actions of citizen scientists utilizing PMIAURP data be regulated and monitored? Are there adequate legal and regulatory mechanisms to address misconduct of citizen scientists, who are not affiliated with the traditional research organizations that usually oversee research?

Other ELSI are related to the complex relationships between the PMI-AURP and the use of information for a participant's medical care. Concerning the incorporation of PMI-AURPgenerated information into clinical records, the following questions might be asked:

- How should clinicians and HPOs address clinical responsibilities and legal liability introduced by the inclusion in medical records of clinically actionable information? How might clinicians' relationships with their patients and with the health-care institutions employing them be altered by the inclusion of information neither ordered by the clinician nor sought by the patient?

- How should physicians or other health-care personnel handle potential inconsistencies in data from different sources (in particular, mHealth results), especially information concerning possibly sensitive issues such as transplant candidate evaluation decisions or psychopharmacological treatment and prescribing? Is there a need for specific procedures to allow all participants to review and correct possible inaccuracies in the data? How will differing claims of accuracy be adjudicated between participants and health-care personnel and between different types of data? 
- How should conflicts between policies of the institutions acting as HPOs and PMI-AURP policies be resolved? What are the implications of patients within an HPO institution-some enrolled in the PMI-AURP and some not-being subject to different policies, such as those governing the return of incidental findings?

- Might participants who enroll through the Direct Volunteer program and those recruited through HPOs be subject to different policies, e.g., policies governing access to data, data security, and return of clinically relevant findings?

- How will privacy protections that apply in the research context-including protections from information seeking by law enforcement agencies-change when information becomes part of the clinical record? How might participants or investigators understand this shift? (Aware that unintentional breaches are not the only event that can compromise privacy, the Precision Medicine Working Group report ${ }^{1}$ suggests the need to protect participants' information from Freedom of Information Act requests and from requests associated with civil or criminal proceedings and to ensure that PMI-AURP information cannot be used to influence participants' eligibility for government benefits. These ideas are not pursued in the funding announcements; however, federal legislation has recently been proposed to strengthen privacy protections for genetic research participants, which might address these concerns. $^{24}$ )

\section{CONCLUSION}

The PMI-AURP promises to improve medical treatment by taking into account individual differences in people's genes, environments, and lifestyles and to introduce a new model of participatory health care that will "transform patients into partners." 3 Reaching these goals will require broad-based, long-term participation of diverse populations. It will also require members of those populations to accept the transformation of their personal medical information into a social good. If the fledgling program evolves as planned, its effects could be pervasive and fundamental, extending broadly to the process and substance of biomedical research as well as to basic assumptions about what constitutes health and illness and how to manage them. The PMI-AURP has anticipated some potential ELSI, as demonstrated by its attention to cohort diversity, security and privacy, and participant engagement. However, as the more comprehensive, if still preliminary, census of ELSI presented here illustrates, the PMI-AURP presents the need for something more. The scope of change that the PMI envisions suggests an obligation to identify and analyze ELSI likely to arise as the PMI-AURP takes shape. It also creates an unprecedented opportunity to study how best to respond to these issues. Although the PMIAURP is still in its formative stage, we call on the PMI to initiate a robust ELSI research program to collect data and conduct normative and empirical analyses to inform the PMI-AURP's efforts.

\section{ACKNOWLEDGMENTS}

We gratefully acknowledge the contributions of the National Human Genome Research Institute's Genomics and Society Working Group (GSWG) (https://www.genome.gov/27551917/thegenomics-and-society-working-group), whose work helped to lay the foundation for this article. The authors are GSWG members. Other GSWG members who contributed include Barbara A. Bernhardt, Perelman School of Medicine, University of Pennsylvania; Wendy K. Chung, Columbia University Medical Center; Gail Henderson, School of Medicine, University of North Carolina; Chanita Hughes-Halbert, Medical University of South Carolina; Amy McGuire, Baylor College of Medicine; Arti K. Rai, School of Law, Duke University; and David Veenstra, School of Pharmacy, University of Washington.

\section{DISCLOSURE}

The authors declare no conflict of interest.

\section{REFERENCES}

1. Precision Medicine Initiative (PMI) Working Group. The Precision Medicine Initiative Cohort Program-building a research foundation for 21st century medicine. 2015. https://www.nih.gov/sites/default/files/research-training/ initiatives/pmi/pmi-working-group-report-20150917-2.pdf. Accessed date 10 June 2015.

2. National Institutes of Health. Frequently asked questions for other transaction awards. Specific to OT-PM-16-001: Cohort Program Direct Volunteer Pilot Studies. 2015. https://www.nih.gov/AllofUs-research-program/frequentlyasked-questions-cooperative-agreements. Accessed June 10, 2016.

3. Collins FS. Preparing to launch the Precision Medicine Initiative Cohort Program. 2016. https://www.nih.gov/about-nih/who-we-are/nih-director/statements/ preparing-launch-precision-medicine-initiative-cohort-program. Accessed June $10,2016$.

4. Williams JS, Walker RJ, Egede LE. Achieving equity in an evolving healthcare system: opportunities and challenges. Am J Med Sci 2016;351:33-43.

5. Budin-Ljøsne I, Harris JR. Ask not what personalized medicine can do for you-ask what you can do for personalized medicine. Public Health Genomics 2015;18:131-138.

6. Joly Y, Saulnier KM, Osien G, Knoppers BM. The ethical framing of personalized medicine. Curr Opin Allergy Clin Immunol 2014;14:404-408.

7. Fiore RN, Goodman KW. Precision medicine ethics: selected issues and developments in next-generation sequencing, clinical oncology, and ethics. Curr Opin Oncol 2016;28:83-87.

8. Chadwick R. Ethical issues in personalized medicine. Drug Discov Today Ther Strateg 2013;10:e171-e174.

9. Faden RR, Kass NE, Goodman SN, Pronovost P, Tunis S, Beauchamp TL. An ethics framework for a learning health care system: a departure from traditional research ethics and clinical ethics. Hastings Cent Rep 2013;Spec No: S16-S27.

10. Adams SA, Petersen C. Precision medicine: opportunities, possibilities, and challenges for patients and providers. J Am Med Inform Assoc 2016;23:787790.

11. Rothstein MA, Wilbanks JT, Brothers KB. Citizen science on your smartphone: an ELSI research agenda. J Law Med Ethics 2015;43:897-903.

12. Cohn EG, Henderson GE, Appelbaum PS; Working Group on Representation and Inclusion in Precision Medicine Studies. Distributive justice, diversity, and inclusion in precision medicine: what will success look like? Genet Med, in press.

13. Armstrong $\mathrm{K}$, Putt $\mathrm{M}$, Halbert $\mathrm{CH}$, et al. Prior experiences of racial discrimination and racial differences in health care system distrust. Med Care 2013;51: 144-150

14. Schwei RJ, Kadunc K, Nguyen AL, Jacobs EA. Impact of sociodemographic factors and previous interactions with the health care system on institutional trust in three racial/ethnic groups. Patient Educ Couns 2014;96:333-338. 


\section{REVIEW}

15. Boulware LE, Cooper LA, Ratner LE, LaVeist TA, Powe NR. Race and trust in the health care system. Public Health Rep 2003;118:358-365.

16. National Institutes of Health. Precision Medicine Initiative Cohort Program Coordinating Center (U2C). Funding Opportunity Number RFA-PM-16-001. http://grants.nih.gov/grants/guide/rfa-files/RFA-PM-16-001.html. June 102015.

17. National Institutes of Health. Precision Medicine Initiative Cohort Program Healthcare Provider Organization Enrollment Centers (UG3/UH3). Funding Opportunity Number RFA-PM-16-002. http://grants.nih.gov/grants/guide/rfafiles/RFA-PM-16-002.html. June 102015.

18. National Institutes of Health. Precision Medicine Initiative Cohort Program Participant Technologies Center (U24). Funding Opportunity Number RFAPM-16-003. http://grants.nih.gov/grants/guide/rfa-files/RFA-PM-16-003.html. June 102015

19. National Institutes of Health. Communication Support for the Precision Medicine Initiative Research Programs at NIH. Funding Opportunity Number OT-PM-16-002. http://www.nih.gov/sites/default/files/research-training/ initiatives/pmi/20151116-pmi-comms-ota-sow.pdf June 102015.
20. Dyer J. Incorporating patient and public input for treatment in comparative effectiveness research. Comparative Effectiveness Research in Health Services. A Levy and B Sobolev (eds.). Springer: New York, 2016. pp. 307-323.

21. Gallivan J, Kovacs Burns K, Bellows M, Eigenseher C. The many faces of patient engagement: does one stand out? J Particip Med 2012;4:e32

22. Kovacs Burns K, Bellows M, Eigenseher C, Gallivan J. 'Practical' resources to support patient and family engagement in healthcare decisions: a scoping review. BMC Health Serv Res 2014;14:175.

23. Kovacs Burns K, Bellows M, Eigenseher C, Jackson K, Gallivan J, Rees J. Exploring patient engagement practices and resources within a health care system: applying a multi-phased mixed methods knowledge mobilization approach. Int J Mult Res Approaches 2014;8:233-247.

24. Senators Warren and Enzi Introduce Bipartisan Bill to Strengthen Genetic Privacy Protections for Research Participants [press release]. Washington, DC: Elizabeth Warren, U.S. Senator for Massachusetts. http://www.warren.senate. gov/?p=press_release\&id=1096. Accessed 5 April 2016 . 